12

\title{
Экспериментальное исследование ускорения электронов при введении расстройки между частотой следования сгустков и частотой возбуждаемого кильватерного поля
}

\author{
(C) А.Ф. Линник, И.Н. Онищенко, В.И. Приступа
}

ННЦ „Харьковский фризико-технический институт“, Харьков, Украина E-mail: onish@kipt.kharkov.ua

\section{Поступило в Редакцию 30 августа 2016 г.}

Проведено экспериментальное исследование возбуждения кильватерных полей в диэлектрической структуре последовательностью сгустков релятивистских электронов и ускорения в них других сгустков той же последовательности при введении расстройки частоты следования сгустков относительно частоты кильватерного поля, возбуждаемого в диэлектрической структуре на черенковском резонансе. Сгустки первой части последовательности возбуждают кильватерную волну, а сгустки второй части последовательности, сдвигаясь в ускоряющую фазу, приобретают дополнительную энергию. Исследована возможность регулировки числа сгустков, возбуждающих кильватерное поле в структуре, и числа последующих ускоряемых сгустков путем изменения величины расстройки.

DOI: 10.21883/PJTF.2017.04.44291.16468

Разработка новых методов высокоградиентного ускорения заряженных частиц вызвана необходимостью уменьшения габаритов коллайдеров, востребованных физикой высоких энергий. Одним из перспектив- 
ных направлений создания будущих линейных коллайдеров является ускорение кильватерным полем, возбуждаемым в диэлектрических структурах электронным сгустком [1-3]. В этой схеме электронный сгусток с большим зарядом (драйвер) при пролете по вакуумному каналу диэлектрической структуры возбуждает продольное кильватерное поле большой амплитуды. С помощью экстрактора СВЧ мощность передается в канал ускорения. Инжектированный с соответствующей задержкой для попадания в ускоряющую фазу кильватерного поля сгусток с малым зарядом (витнес) ускоряется.

В работах $[4,5]$ показана возможность применения вместо одного сгустка-драйвера с большим зарядом последовательности сгустков с суммарным эквивалентным зарядом. В этом случае инжекция ускоряемых сгустков может быть реализована [6] без использования дополнительного ускорителя-инжектора за счет смещения в ускоряющую фазу возбужденного кильватерного поля сгустков задней части последовательности при их задержке во времени отклонением и возвращением на линию возбуждения кильватерного поля сгустками передней части последовательности. В этом методе проблематичным является требуемое быстрое включение отклоняющего магнитного поля. В настоящей работе предложен и экспериментально исследован метод возбуждения кильватерного поля одними сгустками и ускорения других сгустков одной и той же последовательности, не требующий быстро действующих отклоняющих магнитных систем. Суть метода заключается во введении расстройки $\Delta f$ между частотой следования сгустков $f_{m}$ и частотой возбуждаемого кильватерного поля $f_{0}\left(\Delta f=f_{m}-f_{0}\right)$. В отсутствие расстройки $\Delta f=0$ все сгустки последовательности находятся в тормозящей фазе возбуждаемого поля и теряют свою энергию на возбуждение кильватерного поля. При наличии расстройки $\Delta f \neq 0$ сгустки передней части последовательности теряют свою энергию на возбуждение кильватерного поля, а сгустки задней части последовательности, постепенно смещаясь по фазе, попадают в ускоряющее кильватерное поле, возбуждаемое предыдущими сгустками, и приобретают дополнительную энергию. Соответственно кильватерное поле представляет собой биение с периодом $T_{1}=1 / \Delta f$, определяемым величиной расстройки. В результате в течение длительности последовательности сгустков $T$ образуется несколько пар тормозящейся и ускоряемой частей $M=T / T_{1}$. Для точечных сгустков моноэнергетичных электронов число сгустков $N^{*}$

Письма в ЖТФ, 2017, том 43, вып. 4 


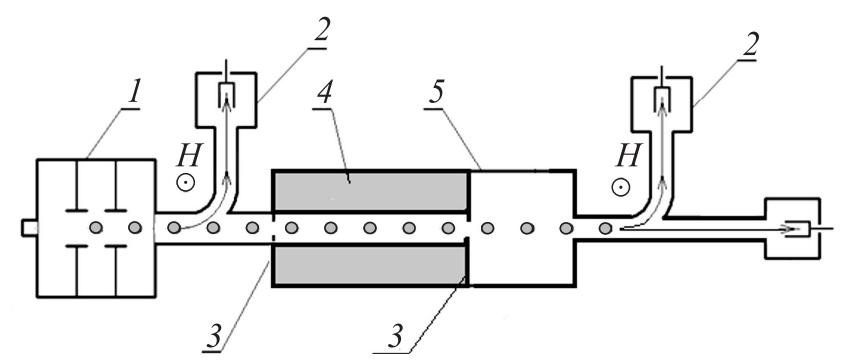

Рис. 1. Схема экспериментальной установки: 1 - ускоритель „Алмаз-2М“, 2 магнитные анализаторы, 3 - входная и выходная диафрагмы, 4 - тефлоновая трубка; 5 - металлический волновод.

первой части последовательности, которые возбуждают кильватерное поле, определяется из условия смещения по фазе $N^{*}$-го сгустка на $\pi$.

Следующая часть последовательности сгустков такой же длительности ускоряется. При этом каждый сгусток ускоряемой части последовательности оказывается в разных фазах и ускоряется разными по величине кильватерными полями. Это приводит к размытому по энергии спектру ускоренных сгустков, что тем не менее позволяет исследовать процесс ускорения сгустков возбужденными кильватерными полями.

Схема экспериментальной установки для исследования возбуждения кильватерного поля в диэлектрической структуре последовательностью сгустков и ускорения в этих полях других сгустков той же последовательности, сдвинутых в ускоряющие фазы за счет введения расстройки между частотой следования сгустков и частотой возбуждаемого кильватерного поля, представлена на рис. 1.

Линейный резонансный электронный ускоритель „Алмаз-2М“ создавал пучок релятивистских электронов, энергию которых можно менять в пределах от 2.5 до $4.8 \mathrm{MeV}$, ток пучка $0.8 \mathrm{~A} \mathrm{в} \mathrm{импульсе}$ длительностью $2 \cdot 10^{-6} \mathrm{~s}$. Каждый импульс представляет собой последовательность $N=6 \cdot 10^{3}$ электронных сгустков, каждый длительностью $6 \cdot 10^{-11} \mathrm{~s}$, зарядом $2.6 \cdot 10^{-10} \mathrm{C}$ и изменяемой частотой их следования $f_{m}$. К ускорителю „Алмаз-2М“ пристыковывалась камера, в которой размещается диэлектрическая структура. Диэлектрическая структура представляет собой медный цилиндрический волновод 5 с внутренним диаметром $85 \cdot 10^{-3} \mathrm{~m}$, заполненный диэлектрической

Письма в ЖТФ, 2017, том 43, вып. 4 
трубкой 4 из фторопласта $\left(\varepsilon=2.04, \operatorname{tg} \delta=4 \cdot 10^{-4}\right)$, внешний диаметр которого равен внутреннему диаметру медного волновода, с каналом диаметром $2.1 \cdot 10^{-2} \mathrm{~m}$ для прохождения электронных сгустков. Длина заполненной диэлектриком части равна $31.9 \cdot 10^{-2} \mathrm{~m}$, что соответствует трем длинам волны основной возбуждаемой моды.

Для выбранных параметров диэлектрической структуры частота кильватерного поля, возбуждаемого сгустками на черенковском резонансе, была фиксированной и равнялась $f_{0}=2805 \mathrm{MHz}$. Расстройка между частотой следования сгустков $f_{m}$ и частотой кильватерного поля $f_{0}$ обеспечивалась изменением частоты следования сгустков $f_{m}$, равной частоте СВЧ-поля клистрона, подаваемого на вход ускоряющей секции ускорителя. Для изменения частоты СВЧ-поля в эксперименте использовался клистрон-усилитель КИУ-12М мощностью до $20 \mathrm{~kW}$ в импульсе $2 \cdot 10^{-6} \mathrm{~s}$ с входным сигналом мощностью до $10 \mathrm{~kW}$ от задающего генератора „Рубин“ на базе магнетрона МИ-30. Изменением частоты сигнала задающего генератора была получена частота следования сгустков, изменяемая в пределах от 2803 до $2807.5 \mathrm{MHz}$.

Энергетические спектры электронов сгустков измерялись с помощью магнитных анализаторов 2, расположенных на входе и выходе диэлектрической структуры.

Экспериментально исследовалось влияние расстройки между частотой, возбуждаемой в структуре кильватерной волны, и частотой следования сгустков последовательности на количество сгустков $N^{*}$, попадающих в тормозящие фазы и возбуждающих кильватерное поле, и такое же количество сгустков, попадающих в ускоряемые фазы. Для расстройки, при которой $N^{*}=N / 2$, имеет место одно биение. В случае $N^{*}<N / 2$ на огибающей СВЧ-кильватерного поля наблюдалось несколько биений.

На рис. 2 представлены экспериментальные и расчетные кривые зависимости $N^{*}$ от величины расстройки $\Delta f$. Кривая 1 (кружки) представляет зависимость экспериментально полученных количеств сгустков $N^{*}$, которые тормозятся (область роста кильватерного поля), и количеств сгустков, которые ускоряются (область уменьшения кильватерного поля), от величины $\Delta f$. Расчет количества сгустков $N^{*}$ по формуле (1) приведен в виде кривой 2 (квадратики). Расчетная и измеренная экспериментально зависимости находятся в удовлетворительном согласии. 


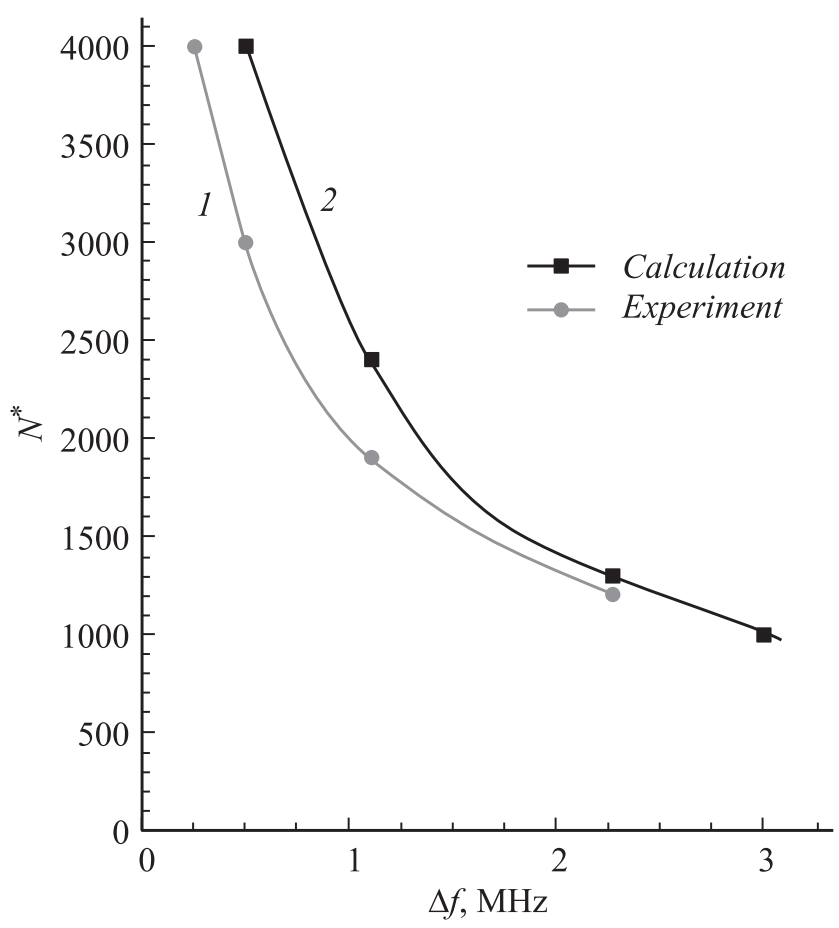

Рис. 2. Зависимость числа сгустков $N^{*}$, возбуждающих кильватерное поле, от величины расстройки $\Delta f=f_{m}-f_{0}$.

Энергетические спектры электронов пучка, измеренные с помощью магнитных анализаторов, установленных на выходе ускорителя и на выходе диэлектрической структуры, представлены на рис. 3: квадратики исходные энергетические спектры; кружки - энергетические спектры электронов после возбуждения кильватерного поля и взаимодействия с ним сгустков. На рис. $3, a$ показан резонансный случай (нулевая расстройка $\Delta f=0$ ), на рис. $3, b-$ нерезонансный случай (ненулевая расстройка $\Delta f=2.5 \mathrm{MHz}$ ).

Из рис. $3, a$ следует, что при наличии диэлектрика в случае резонанса весь энергетический спектр смещается на $400 \mathrm{keV}$ как целое

Письма в ЖТФ, 2017, том 43, вып. 4 

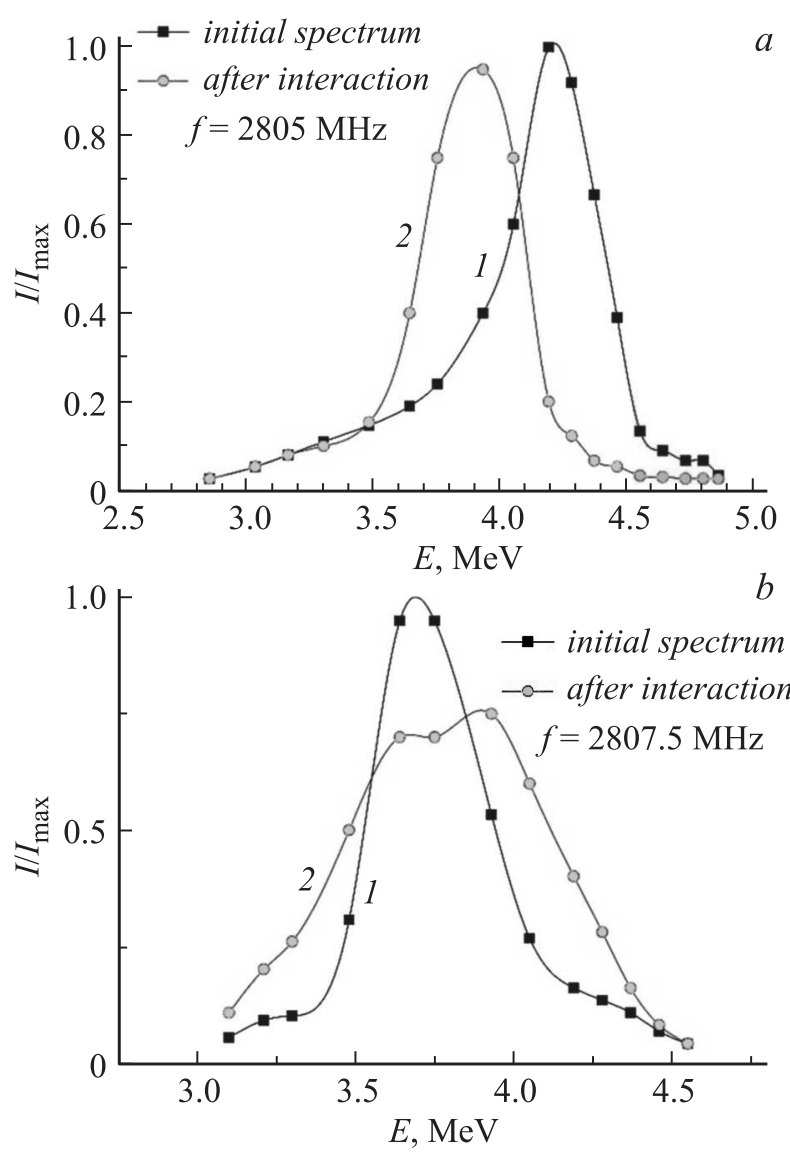

Рис. 3. Энергетические спектры электронов сгустков, инжектируемых из ускорителя „Алмаз-2М““ (кривая 1) и провзаимодействовавших с диэлектрической структурой (кривая 2): $a-\Delta f=0 ; b-\Delta f=f_{m}-f_{0}=2.5 \mathrm{MHz}$.

в область меньших энергий, что объясняется потерей энергии на возбуждение колебаний всех сгустков, как резонансных.

При наличии расстройки между частотой следования сгустков и частотой кильватерного поля $\Delta f=f_{m}-f_{0}=2.4 \mathrm{MHz}$ (рис. $3, b$ ) в энергетических спектрах электронов наблюдаются как потерявшие

Письма в ЖТФ, 2017, том 43, вып. 4 
энергию электроны $(-150 \mathrm{keV})$, так и ускоренные до энергии выше первоначальной $(+150 \mathrm{keV})$. Это свидетельствует о том, что часть сгустков последовательности, смещаясь по фазе, попадает в ускоряющую фазу кильватерной волны, возбуждаемой предыдущими сгустками той же последовательности, и отбирает энергию кильватерного поля.

Изменением величины расстройки можно регулировать число сгустков последовательности, которые возбуждают кильватерную волну, и число последующих сгустков, которые попадают в ускоряющую фазу кильватерного поля и получают дополнительную энергию. При увеличении расстройки возникают условия, когда появляется биение кильватерного поля с несколькими частями последовательности заторможенных и ускоренных сгустков.

Таким образом, экспериментально продемонстрирована возможность использовать расстройку частоты следования сгустков относительно частоты черенковского кильватерного поля, возбуждаемого в диэлектрической структуре, для ускорения части сгустков последовательности, без необходимости использовать отдельный ускорительинжектор.

Работа поддержана программой „Global Initiatives for Proliferation Prevention (GIPP)“،, проект ANL-T2-247-UA (УНТЦ № P522).

\section{Список литературы}

[1] Gai W., Schoessow P. // Nucl. Instr. Meth. Phys. Res. A. 2001. V. 459. N 1. P. 1-5.

[2] Onishchenko I.N., Sidorenko D.Yu., Sotnikov G.V. // Phys. Rev. E. 2002. V. 65. P. 066501 (1-11).

[3] Батурин С.С., Шейнман И.Л., Альтмарк А.М. // Письма в ЖТФ. 2011. Т. 37. Вып. 9. С. 7-13.

[4] Zhang T.-B., Hirshfield J.L., Marshal T.C., Hafizi B. // Phys. Rev. E. 1997. V. 56. N 4. P. 4647-4655.

[5] Onishchenko I.N., Kiselev V.A., Linnik A.F., Sotnikov G.V. // Proc. IPAC'13. Shanghai, China, 12-17 May, 2013. P. 1259-1261.

[6] Вишняков В.А, Закутин В.В., Кушнир В.А. и др. // Труды 10-го Всесоюзного совещания по ускорителям заряженных частиц. Дубна, Россия, 21-23 октября 1986 г. Т. 1. С. 257-259.

Письма в ЖТФ, 2017, том 43, вып. 4 